\title{
Discusiones
}

\section{¿Abandona Leibniz la concepción del espacio como lugar universal de las cosas después de 1671? Observaciones críticas al artículo de Federico Raffo Quintana \\ [Does Leibniz Abandon the Conception of Space as the Universal Place of Things after 1671? Critical Remarks to Federico Raffo Quintana's Paper]}

\author{
CAMILO SILVA \\ École Normale Supérieure de Paris-Université Paris Sciences et Lettres \\ Groupe Mathesis-République des Savoirs \\ camilo.silva@ens.fr, camilof.silva@gmail.com
}

\begin{abstract}
Resumen: En la siguiente discusión presento algunas objeciones al artículo de Federico Raffo Quintana “La noción de 'espacio' en los escritos juveniles de Leibniz". ${ }^{1}$ Contra la interpretación de Raffo, quien considera que la concepción del espacio como lugar universal de las cosas es una idea que Leibniz abandona de manera muy temprana —según el autor en 1671—, intento mostrar que esta concepción trasciende sin duda el periodo de los escritos juveniles de Leibniz y que ciertas confusiones conceptuales en la lectura de Raffo hacen que, en vez de ver una armónica continuidad en la teoría del espacio de Leibniz, vea en ella una ruptura en su evolución histórica, a la que, además, asocia algunas dicotomías discutibles o espurias.
\end{abstract}

Palabras clave: espacio absoluto, espacio abstracto, extensión, relacionismo, sustancialismo

\begin{abstract}
In this paper I introduce objections to Federico Raffo Quintana's paper "The Notion of 'Space' in the Young Leibniz Writings". Against Raffo, who considers that the Leibnizian conception of space as the universal place of things is an idea that Leibniz early abandons — Raffo says in 1671-, I will try to show that such a conception goes beyond the Leibniz's young writings and that it is because of some conceptual confusions in Raffo's survey that he, instead of seeing a harmonic continuity in Leibniz's theory of space, sees a rupture in its historical evolution, associating to it some disputable or spurious dichotomies.
\end{abstract}

Key words: absolute space, abstract space, extension, relationism, substantivalism

${ }^{1}$ Diánoia, vol. 62, no. 78, 2017, pp. 75-97.

Revista de Filosofía Diánoia, vol. 64, no. 83 (noviembre de 2019-abril de 2020): pp. 133-151

e-ISSN: 1870-4913 • DOI: https://doi.org/10.22201/iifs.18704913e.2019.83.1718 
Introducción. Motivación de la discusión

En "La noción de 'espacio' en los escritos juveniles de Leibniz", ${ }^{2}$ Federico Raffo Quintana ofrece un estudio minucioso de la concepción del espacio en el periodo juvenil de Leibniz. Pese al valioso y acabado análisis de Raffo, encuentro en él algunas dificultades exegéticas que, por el interés e importancia que reviste su trabajo, vale la pena discutir.

A partir de un pasaje en la correspondencia entre Thomasius y Leibniz, Raffo atribuye a este último una concepción sustancialista del espacio, que asigna a la primera de las tres etapas que distingue. ${ }^{3}$ Esta atribución de Raffo es correcta porque el mismo Leibniz define así la naturaleza del espacio. Raffo evoca sólo el primero de los dos argumentos que, en su carta del 6 de octubre de 1668 dirigida a Thomasius, Leibniz formula para probar esta tesis. ${ }^{4}$ En clave silogística, Leibniz explica que, si la geometría es una ciencia, y si toda ciencia tiene por objeto una sustancia, entonces el espacio - objeto de la geometría- debe ser una sustancia. ${ }^{5}$ Sin embargo, el filósofo alemán elabora un segundo argumento que, con base en el experimento mental de la supresión de los cuerpos en el espacio, le permite inferir la prioridad ontológica que éste mantiene con respecto a aquéllos. En palabras de Leibniz:

El espacio [...] es más sustancial [substantialius] que el cuerpo mismo. En efecto, una vez supreso [sublato] el cuerpo, si ningún otro [cuerpo] viene a ocupar su lugar, permanece tanto el espacio como su dimensión, lo que se denomina vacío [vacuum], mientras que, en cambio, una vez supreso [sublato] el espacio, ya no hay cuerpo. ${ }^{6}$

Al contrastar la hipótesis de la supresión del espacio con la de la supresión de los cuerpos, Leibniz infiere la sustancialidad que cabe atribuir al espacio a partir de la prioridad ontológica que éste, por asimetría,

${ }^{2}$ Raffo 2017.

${ }^{3}$ Cfr. Raffo 2017, pp. 75-76.

${ }^{4}$ Cfr. Raffo 2017, p. 78.

${ }^{5}$ Cfr. Leibniz a Thomasius, en Sämtliche Schriften und Briefe 1923-, serie II, vol. 1, p. 11. De acuerdo con el empleo estándar de las referencias a las obras de Leibniz, abreviaré dicha edición como A, seguido de la serie, volumen y página. Asimismo, emplearé las siguientes abreviaciones: GM = Mathematische Schriften, 1849-1863, y GP = Philosophischen Schriften, 1875-1890, seguidos del tomo y página, y $\mathrm{R}=$ Correspondance Leibniz-Clarke, 1957, seguido de la página. La traducción de los textos que cito en este artículo es mía, a menos que se indique lo contrario.

${ }^{6}$ Leibniz a Thomasius, A II, 1, 11.

Revista de Filosofía Diánoia, vol. 64, no. 83 (noviembre de 2019-abril de 2020) e-ISSN: 1870-4913 • DOI: https://doi.org/10.22201/iifs.18704913e.2019.83.1718 
mantiene con respecto a aquéllos. El argumento no carece de interés, ya que, pese a que a partir de sus premisas Leibniz infiere aquí una concepción sustancialista del espacio - en concordancia con el primer argumento que evoca Raffo-, el mismo argumento constituye una versión primigenia de la concepción que, en lo sucesivo, Leibniz defenderá: la prioridad del espacio con respecto a los cuerpos es una característica (relativa) que lo define. ${ }^{7}$

Lo que destaca en este segundo argumento es que la prioridad del espacio en relación con los cuerpos implica una concepción receptacular del espacio, la cual, en la terminología propia de Leibniz, equivale a lo que él llama "el lugar universal de las cosas". ${ }^{8}$ En este sentido, pese a que Leibniz abandona pronto la tesis de la sustancialidad del espacio, la asimetría ontológica entre éste y los cuerpos no es una tesis a la que renuncie, de modo que afirmar que Leibniz no hace valer la concepción del espacio como lugar universal de las cosas después de 1671 — como Raffo, sin embargo, sostiene -9 es contestable. En rigor, la evolución de la concepción del espacio de Leibniz —ya en el periodo de sus escritos de juventud- no remonta a un abandono de la concepción receptacular - entendida como lugar universal de las cosas-, sino a una transmutación categorial del concepto de espacio, lo que afecta su estatus ontológico simpliciter, no así la prioridad ontológica que goza en relación con los cuerpos. El problema parece radicar en que Raffo no distingue la tesis de la receptacularidad de la tesis del sustancialismo, pues, según él, la tesis del espacio como "lugar universal' podría entenderse en términos sustancialistas". ${ }^{10}$

A pesar de aceptar que — como explica Raffo—, ${ }^{11}$ debido al dinamismo que Leibniz atribuye a las sustancias, el espacio, supuesta su inmovilidad, no puede caracterizarse como una entidad sustancial, no resulta claro en qué sentido o por qué esta restricción categorial im-

${ }^{7}$ Cfr. también Leibniz a Thomasius, 20/30 abril de 1669, A II, 1, 21-22.

${ }^{8}$ Leibniz a Thomasius, A II, 1, 21-22. En otros escritos contemporáneos, Leibniz insiste en la tesis de la receptacularidad del espacio sobre la cual descansa la caracterización de éste como lugar universal de las cosas. Cfr. por ejemplo, Confessio naturae contra atheistas (1668-1669), A VI, 1, 490 y 493; Demonstratio possibilitatis mysteriorum eucharistiae, Refutatio hypotheseos Thomae Angli (1668 (?)), A VI, 1, 508; De rationibus motus (1669-1670 (?), tercer borrador) , A VI, 2, 167-170; Dissertatio praeliminaris Marii Nizolii De veris principiis et vera ratione philosophandi libri IV (1670), A VI, 2, 443; Specimen demonstrationum de Natura Rerum Corporearum ex phaenomenis (1671 (?), segundo borrador), A VI, 2, 304-308.

${ }^{9}$ Cfr. Raffo 2017, p. 76.

${ }^{10}$ Raffo 2017, p. 76.

${ }^{11}$ Cfr. Raffo 2017, pp. 81-87.

Revista de Filosofía Diánoia, vol. 64, no. 83 (noviembre de 2019-abril de 2020) e-ISSN: 1870-4913 • DOI: https://doi.org/10.22201/iifs.18704913e.2019.83.1718 
plica una incompatibilidad —según piensa Raffo—— ${ }^{12}$ con la concepción del espacio como lugar universal de las cosas: es posible definir el espacio como un receptáculo o lugar universal de las cosas y, al mismo tiempo, como un orden o relación de coexistencia entre los cuerpos. De hecho, según veremos, es justo esta conciliación entre ambas caracterizaciones el objeto central que guía las reflexiones de Leibniz sobre el concepto de espacio.

La crítica que me propongo desarrollar en relación con la interpretación de Raffo pone de relieve en particular las siguientes tres tesis: a) Leibniz no abandona, después de 1671, una concepción del espacio como lugar universal de las cosas, pues, de manera invariable, concibe el espacio como un receptáculo de los cuerpos; b) no existe ninguna incompatibilidad entre - lo que Raffo llama- ${ }^{13}$ una concepción estática y una concepción dinámica del espacio, y c) de hecho, la concepción estática-receptacular del espacio, cuerpo matemático o lugar universal de las cosas equivale a lo que Leibniz, en su madurez, denomina en sus escritos matemáticos "espacio absoluto" o, como prefiere en la correspondencia con Clarke, "espacio abstracto", mientras que, por su parte, la concepción dinámica-relacional equivale al "espacio actual" o "espacio real" que define como el orden entre los cuerpos materiales coexistentes.

1. Leibniz y la concepción receptacular del espacio como lugar universal de las cosas. Escritos de 1680-1690

Ciertos escritos de Leibniz atestiguan que no abandona después de 1671 una concepción del espacio como el lugar universal de las cosas. En efecto, si a lo largo de la década de 1670 Leibniz define todavía el espacio como todos los lugares ${ }^{14} \mathrm{o}$, de manera más amplia, como una "entidad" equivalente a un receptáculo, continente o lugar de los cuerpos, ${ }^{15}$ y que representa, por consiguiente, una condición de éstos, ${ }^{16}$ a

${ }^{12}$ Cfr. Raffo 2017, pp. 84-87.

${ }^{13}$ Cfr. Raffo 2017, p. 96.

${ }^{14}$ Cfr. De origine rerum ex formis (1676 (?)), A VI, 3, 519.

${ }^{15}$ Cfr. De magnitude (1676 (?)), A VI, 3, 484; Communicata ex literis Domini Schulleri (1676 (?)), A VI, 3, 282, nota 24; Über Spinozas Ethik (1675-1676 (?)), A VI, 3, 385 y Demonstratio axiomatum Euclidis (1679), A VI, 4-A, 179.

${ }^{16}$ Cfr. Definitiones cogitationesque metaphysicae (1678-1680/1 (?)), A VI, 4-B, 1397; Specimen demonstrationum De Natura Rerum corporearum ex phaenomenis, A VI, 2, 305 y De veritatibus, de mente, de Deo, de universo (1676), A VI, 3, 511.

Revista de Filosofía Diánoia, vol. 64, no. 83 (noviembre de 2019-abril de 2020) e-ISSN: 1870-4913 • DOI: https://doi.org/10.22201/iifs.18704913e.2019.83.1718 
partir de 1680, por medio de diversas fórmulas equivalentes, el filósofo alemán insiste en sostener la misma tesis.

Al menos en tres opúsculos del decenio de 1680, Leibniz concibe el espacio en estos términos. En uno de ellos (1683 (?)), sostiene que "todas las cosas de la naturaleza existen [...] [en] el sustrato receptacular último [substratum receptorium ultimum] que se denomina espacio". ${ }^{17}$ En otro texto (1684-1685/6 (?)), considera que "el tiempo y el lugar, o sea el espacio, son receptáculos [receptacula] [que] acogen [recepta] los cuerpos que existen en ellos". ${ }^{18}$ Por último, en un tercer opúsculo (1685-1687 (?)), define el espacio universal [spatium mundanum] como "aquello que es extenso, y aquello en lo que está todo lo que es extenso". ${ }^{19}$

Aunque al distinguir las funciones asignables a los cuerpos y al espacio a partir de la relación "estar en" Leibniz no atribuye en forma explícita a este último una prioridad con respecto a aquéllos, es evidente que su razonamiento apunta a destacar esa asimetría toda vez que el continente no puede depender o constituirse a partir de lo contenido: que los cuerpos se den en el espacio implica ( $a$ priori) que no pueden darse fuera o más allá de éste. En otros términos, al ser anterior (prius natura) a los cuerpos, el espacio es una especie de receptáculo en el que los cuerpos pueden situarse y que corresponde a su lugar universal. ${ }^{20}$

La concepción del espacio como lugar universal de las cosas no es propuesta por Leibniz sólo en esta época: reaparece, bajo diversas variantes, en otros escritos. Por ejemplo, desde una perspectiva teológica ligada al problema de la creación, en De rerum originatione radicali (1697), Leibniz pregona de manera implícita esta concepción al aseverar:

El tiempo y el lugar [locus] o, como pueden denominarse, la capacidad o receptividad del mundo [receptitas vel capacitas mundi] pueden considerarse como el dispendio o terreno [sumtu sive terreno] en el cual pueden edificarse de la manera más adecuada [in quo quam commodissime est aedificandum] las variedades de las formas que corresponden a la comodidad

${ }^{17}$ Universum corpus pansophicum, A VI, 4-B, 1171. Las cursivas son del original.

${ }^{18}$ De mundo praesenti, A VI, 4-B, 1509.

${ }^{19}$ De extenso, spatio, corpore et puncto (primera versión), A VI, 4-A, 668.

${ }^{20}$ Grosso modo y leída a través de un prisma casi kantiano, ésta es la tesis implícita que defiende y desarrolla de manera exhaustiva y sólida Gueroult 1982, a cuya interpretación mi lectura se adosa en lo sucesivo. Agradezco la observación que uno de los árbitros me ofreció en una versión preliminar de este artículo a propósito del artículo de Gueroult, cuya proximidad y relevancia teóricas en relación con la interpretación que aquí defiendo no había sido objeto de mi atención.

Revista de Filosofía Diánoia, vol. 64, no. 83 (noviembre de 2019-abril de 2020) e-ISSN: 1870-4913 • DOI: https://doi.org/10.22201/iifs.18704913e.2019.83.1718 
del edificio, y a la multitud y elegancia de las habitaciones. Y esto ocurre tal como en ciertos juegos [ludis] en los que todos los espacios [loca] de la tabla son llenados [replenda] según ciertas reglas [legis], sin cuya habilidad [artificio] quedarán espacios desiguales excluidos [spatiis exclusus iniquis] [...] [Pero] lo que existe es [aquello que es] máximamente posible con respecto al tiempo y al lugar (es decir, con respecto al orden de posibilidad de los coexistentes). ${ }^{21}$

El lugar, entendido como la receptividad o capacidad del mundo, no puede concebirse, en este plano teológico que figura la creación, como un mero orden relacional contingente: el lugar o espacio es una condición de posibilidad de la coexistencia de los cuerpos, es decir, el lugar universal en el que éstos pueden situarse. En rigor, el espacio no es un orden entre los cuerpos coexistentes, sino su posibilidad de coexistencia. A la luz de este pasaje, es evidente que, para Leibniz, el espacio trasciende y precede ontológicamente a los cuerpos.

2. La concepción leibniziana de un espacio abstracto como receptáculo universal y condición de posibilidad de los cuerpos. Textos metafísicos tardíos

Además de los fragmentos anteriores, existen más pruebas en favor de la asimilación de una concepción del espacio como lugar universal de las cosas en la madurez de Leibniz. En efecto, el filósofo establece esta tesis en dos dominios teóricos distintos, aunque complementarios, a saber, la metafísica y la geometría. En esta sección me concentraré en el análisis de sus textos metafísicos y reservaré la siguiente a una exposición del modo en que dicha concepción aparece en algunos de sus escritos tardíos de geometría.

El análisis del concepto de espacio en los escritos metafísicos tardíos de Leibniz reviste una complejidad especial no sólo porque es el resultado de una integración de supuestos y principios misceláneos — por ejemplo, ontológicos, epistemológicos y teológicos-, sino también porque la literalidad cede su lugar a la interpretación. La comprensión de los pasajes de los escritos metafísicos que a continuación abordaré depende, en este sentido, de un análisis más fino, pese a lo cual permiten, mutatis mutandis, atribuir a Leibniz, de manera relativamente incontestable, una concepción del espacio en los términos que he sugerido.

En el inicio de su artículo, Raffo cita dos afirmaciones de una carta de Leibniz a Clarke, en la primera de las cuales Leibniz declara que

${ }^{21}$ GP VII, 303-304. 
"no hay espacio donde no hay materia" 22 y, en la segunda, que "el espacio mismo no es una realidad absoluta". ${ }^{23} \mathrm{Si}$ bien ambas apuntan a criticar la concepción newtoniana del espacio, poseen un alcance distinto, se fundan sobre premisas heterogéneas y tienen por objeto rebatir dos tesis completamente diferentes. En efecto, si la segunda de estas aseveraciones tiene por objeto criticar la naturaleza sustancial que, de acuerdo con Leibniz, supone la concepción newtoniana del espacio, la primera pretende negar la posibilidad de un espacio real o actual en el que no haya materia, pero sólo porque, contingentemente, no existe el vacío. ${ }^{24}$ En otras palabras, si la segunda afirmación critica la posibilidad

${ }^{22}$ Leibniz' fünftes Schreiben, § 62, GP VII, 406 (cit. en Raffo 2017, p. 76).

${ }^{23}$ Leibniz' fünftes Schreiben, § 62, GP VII, 406 (cit. en Raffo 2017, p. 76).

${ }^{24}$ Esta tesis puede inferirse, por ejemplo, de la carta de Leibniz a Bernoulli, 13/23 de enero de 1699, GP III, 565, así como de dos cartas de Leibniz à la princesse de Galles, del 12 de mayo de 1716 y del 2 de junio de 1716 (cfr. R, 76-77 y 7879 respectivamente). Sin embargo - y tal como un árbitro me ha hecho ver-, la cuestión no deja de ser discutible y delicada. Si bien en ciertos escritos - como a los que recién me referí- Leibniz parece considerar la inexistencia del vacío como contingente por ser incompatible tanto con la experiencia como también con la perfección de Dios, en otros parece negar la posibilidad de que el vacío, sin dejar de ser intrínsecamente posible, sea consistente de manera relativa a la existencia ya que, al ser indiscernibles entre sí, las partes del vacío no pueden, en principio, ser objeto de la elección de Dios, quien carecería en este escenario de una razón suficiente que determine o motive su acción (cfr. por ejemplo, Principia logico-metaphysica (1689 (?), A VI, 4-B, 1647). Si bien en este segundo sentido el vacío no resulta imposible en términos metafísicos, parece ser inactualizable: si los principios de razón suficiente y de identidad de los indiscernibles son principios necesarios relativos a la creación o actualización de un mundo posible cualquiera, el vacío sería inconsistente con la existencia. Ahora bien, supuesta la distinción entre estas dos tesis que niegan la hipótesis del vacío, a saber, la tesis débil o contingente de la inexistencia del vacío (TDCI) y la tesis fuerte o modal de la imposibilidad de existencia del vacío (TFMI), es preciso indicar que la segunda entraña una dificultad, a saber, distinguir entre posibilidad (o mundos posibles) y actualizabilidad (o mundos actualizables). Pese a que, de manera intuitiva, TFMI me parezca correcta, dado que encierra diversas dificultades y exige una justificación aparte que aquí no puedo proporcionar, me remito a considerar la primera como suficientemente adecuada para efectos de mi discusión, por cuanto 1) si bien TDCI puede entenderse como una implicación trivial de TFMI, TDCI parece contar con un soporte filosófico propio e independiente, y 2) TDCI parece, en este sentido, menos problemática en términos filosóficos. Aunque no necesariamente desde esta óptica modal, la cuestión del estatus, los fundamentos y las consecuencias de la tesis leibniziana de la inexistencia del vacío ligada al principio de identidad de los indiscernibles ha sido muy discutida por diversos estudiosos (cfr. por ejemplo, Rodriguez-Pereyra 2014 y Ruiz 2014, pp. 417-425). Para una discusión análoga a la distinción entre TDCI y TFMI relativa

Revista de Filosofía Diánoia, vol. 64, no. 83 (noviembre de 2019-abril de 2020) e-ISSN: 1870-4913 • DOI: https://doi.org/10.22201/iifs.18704913e.2019.83.1718 
de la sustancialidad del espacio, la primera rebate como falsa la tesis de un espacio que, desprovisto de materia, sea considerado como real: la primera tesis niega el estatus sustancial del espacio, mientras que la segunda niega la existencia del vacío en el espacio actual. En concordancia con su propósito, Raffo no comenta estas afirmaciones: limitándose sólo a mencionarlas, no las distingue. En esta confusión o indistinción radica, a mi juicio, el punto problemático de su interpretación.

Lo que puede explicar que Raffo considere que Leibniz no sostiene una concepción del espacio como lugar universal de las cosas después de 1671 es, tal vez, que no distingue las dos acepciones que el concepto "real" tiene en ambos enunciados: es probable, pues, que Raffo considere que el espacio no puede ser un lugar universal de las cosas $s i$, en conformidad con el relacionismo, son las relaciones entre las cosas materiales las que - por decirlo así- fundan el espacio, pues — dicho de otro modo- si el espacio se define por las relaciones de coexistencia entre los cuerpos, entonces presuponer un espacio (absoluto) fuera de tales relaciones es o innecesario o absurdo. ${ }^{25}$ En consecuencia, es al parecer porque la presuposición de un lugar universal de las cosas es prima facie irreconciliable con la definición del espacio como un orden de coexistencia entre los cuerpos que Raffo asegura que la idea de un lugar universal queda -o debe quedar- excluida si la teoría del espacio de Leibniz aspira a ser consistente.

En los escritos tardíos de Leibniz la idea del espacio como lugar universal de las cosas constituye una pieza clave para la comprensión de su metafísica del espacio. El problema - que al parecer no atiende Raffodescansa en que, con la relatividad o relacionismo espacial, Leibniz designa dos cosas distintas, a saber, el espacio como orden de coexistencia entre los cuerpos y el espacio como posibilidad de coexistencia de éstos: el primero corresponde al espacio real, definido por las relaciones entre los cuerpos, y es puramente contingente; en cambio, el segundo corresponde al espacio ideal, definido no como orden entre los coexistentes actuales, sino como orden de coexistencia que, al ser de la naturaleza de las verdades eternas, conlleva una necesidad metafísica. ${ }^{26}$

a la imposibilidad o inactualizabilidad del espacio absoluto newtoniano, cfr. Silva 2018, en particular p. 36, n. 34.

${ }^{25} \mathrm{Tal}$ es precisamente la interpretación de Winterbourne 1982, pp. 203 y 208, quien considera que Leibniz prescinde del concepto de espacio absoluto para la elaboración de su teoría.

${ }^{26}$ Es la caracterización que propone De Risi 2007, pp. 551 y ss. Aunque por medio de una terminología diferente, Gueroult 1982, pp. 284 y ss., distingue también ambos conceptos. De manera análoga, aunque con otros términos y otras premisas

Revista de Filosofía Diánoia, vol. 64, no. 83 (noviembre de 2019-abril de 2020) e-ISSN: 1870-4913 • DOI: https://doi.org/10.22201/iifs.18704913e.2019.83.1718 
Si al negar que el espacio sea una realidad absoluta Leibniz contrapone la concepción sustancialista del espacio —que, al atribuirla a Newton, juzga imposible o contradictoria- ${ }^{27}$ con su propia concepción relacional tout court, ese mismo relacionismo tout court, referido ahora a su propia concepción del espacio, debe someterse a una descomposición analítica pues Leibniz, desde una óptica modal, distingue dos tipos de relaciones espaciales, a saber, las determinadas por entidades reales o actuales y sobre las que se funda el espacio - que él denominareal o actual, y las relaciones que, sin estar determinadas por entidades reales o actuales, se definen por relaciones puramente abstractas o ideales, y que corresponden por consiguiente al espacio abstracto o ideal que, por su idealidad, precede ontológicamente y es independiente del espacio real. ${ }^{28}$ Explicaré en detalle esta sutil y decisiva distinción y las consecuencias que de ella se desprenden.

Las dos afirmaciones que cita Raffo aparecen en el § 62 del Quinto escrito de Leibniz a Clarke que, por las dificultades analíticas que mencioné, exige que se reproduzca íntegramente. En respuesta a la acusación de una presunta identidad entre espacio y materia que Clarke imputa a Leibniz, ${ }^{29}$ éste explica:

Yo no digo que el espacio y la materia sean lo mismo; digo solamente que no hay espacio donde no hay materia, y que el espacio en sí mismo no es una realidad absoluta. El espacio y la materia difieren como el tiempo y el movimiento. Sin embargo, aunque diferentes, estas cosas [el espacio y la materia] no pueden darse una sin la otra [se trouvent inséparables]. ${ }^{30}$

La relación que Leibniz establece aquí entre el espacio y la materia es la llave maestra para comprender su concepción del espacio. En rigor, Leibniz sostiene que, pese a que no existe espacio sin materia, ni ma-

de las que deduce consecuencias hasta cierto punto incompatibles con mi análisis, Vailati 1997, p. 116, sugiere la misma distinción.

${ }^{27}$ Cfr. Leibniz' viertes Schreiben, § 16, GP VII, 374.

${ }^{28}$ Distinción que corresponde a —según la terminología de Gueroult 1982, p. 284- lo extenso (extensum) y la extensión (extensio). Cfr. también Ruiz 2014, pp. 339-357.

${ }^{29}$ Cfr. Clarke's vierte Entgegnung, § 15, GP VII, 384-385.

${ }^{30}$ Leibniz' fünftes Schreiben, § 62, GP VII, 406. De aquí en adelante, actualizaré las palabras del francés en las citas cuya ortografía no corresponda a las reglas del francés moderno.

Revista de Filosofía Diánoia, vol. 64, no. 83 (noviembre de 2019-abril de 2020) e-ISSN: 1870-4913 • DOI: https://doi.org/10.22201/iifs.18704913e.2019.83.1718 
teria sin espacio, ambos difieren: ${ }^{31}$ el hecho de que no haya espacio donde no hay materia es estrictamente accidental o contingente. ${ }^{32} \mathrm{De}$ manera más precisa, es porque - como sostiene Leibniz un poco antes en la misma carta- "no hay espacio real fuera del universo material"33 que la materia y el espacio, sin ser idénticos, coinciden. Pero, eo ipso, no es sino en cuanto que real que el espacio coincide con la materia: espacio y materia son esencialmente distintos pero, en cuanto que existentes, son inseparables y coinciden dada la inexistencia del vacío material; ${ }^{34}$ a contrario, sólo si hubiese vacío material, la extensión espacial no coincidiría con la extensión de la materia, lo que, sin embargo, en el mundo actual o contingente no ocurre, supuesta la plenitud. ${ }^{35}$ Por ello, aunque falsa en un sentido contingente, la hipótesis del vacío es perfectamente concebible. ${ }^{36}$

Con el objeto de refrendar la prioridad del espacio en relación con los cuerpos sobre la base de dos escenarios contrafácticos antisimétricos, Leibniz opone la posibilidad del espacio sin cuerpos a la de los cuerpos sin espacio, y afirma que "Dios, al destruir la extensión, destruiría < por ello mismo> los cuerpos; pero no produciendo más que extensión, tal vez, Él no produciría sino sólo el espacio sin cuerpos". ${ }^{37}$ Mediante la contraposición de las hipótesis de la destrucción y producción de la extensión, Leibniz supone una prioridad e independencia

31 “[A] unque distingo la noción de extensión de la de cuerpo, no dejo de creer que no hay vacío, e incluso que no hay ninguna sustancia que pueda llamarse espacio" (Entretien de Philarete et d'Ariste, GP VI, 585). Cfr. también Nouveaux Essais sur l'entendement humain, II, xiii, § 21, A VI, 6, 151.

${ }^{32}$ De acuerdo con la interpretación que sostiene que la inexistencia del vacío es contingente y que ha sido justificada previamente. Cfr. supra n. 24.

${ }^{33}$ Leibniz' fünftes Schreiben, § 29, GP VII, 395-396 (las cursivas son mías). Ésta es la razón por la cual, para Leibniz, "el espacio fuera del mundo es imaginario" (Leibniz' viertes Schreiben, § 7, GP VII, 372).

${ }^{34}$ Además de los pasajes reproducidos antes, cfr. Leibniz a De Volder, 27 de diciembre de 1701, GP II, 234 y Nouveaux Essais sur l'entendement humain, II, iv, § 5, A VI, 6, 127.

${ }^{35}$ Cfr. Leibniz à la princesse de Galles, 12 de mayo de 1716, R, 76-77.

${ }^{36}$ Desde una perspectiva epistemológica, Leibniz explica esto a través del ejemplo de la medición de una esfera vacía. Cfr. Nouveaux Essais sur l'entendement humain, II, xv, § 11, A VI, 6, 155. Sobre este asunto, cfr. los comentarios de Gueroult 1982, pp. 293 y ss.

${ }^{37}$ Entretien de Philarete et d'Ariste, GP VI, 581. En su juventud, Leibniz elaboró un argumento alternativo — de cuño trascendental, basado en la percepción- para probar esta misma asimetría (cfr. Specimen demonstrationum de natura rerum corporearum ex phaenomenis, A VI, 2, 305). Raffo traduce (parcialmente) y comenta este argumento (cfr. Raffo 2017, p. 79).

Revista de Filosofía Diánoia, vol. 64, no. 83 (noviembre de 2019-abril de 2020) e-ISSN: 1870-4913 • DOI: https://doi.org/10.22201/iifs.18704913e.2019.83.1718 
ontológicas del espacio respecto de los cuerpos. Y es quizá con esta asimetría entre espacio y materia que advierte una convergencia entre la tesis que él, en su carta a Thomasius del 6 de octubre de $1668,{ }^{38}$ infiere en su juventud y la posición de Newton, para quien el espacio absoluto sí es una entidad real, esto es —según las categorías de Leibniz-, una sustancia. Ésta parece ser la razón por la cual Leibniz, quizá para prevenir una réplica de Newton-Clarke, se anticipa a la tesis que podría inferirse de la distinción entre materia y espacio al añadir una restricción categorial: por mucho que la extensión del espacio pudiese rebasar la extensión de la materia, no debe por ello considerarse que el "espacio <sea> una realidad absoluta". La crítica de Leibniz a Newton-Clarke se reduce paradójicamente a bloquear la consecuencia que él mismo había deducido casi cincuenta años atrás: la prioridad ontológica del espacio con respecto a los cuerpos no debe considerarse una condición suficiente para concebirlo como real, es decir, como una entidad sustancial. El espacio separado de la materia o de los cuerpos materiales sí es un receptáculo y, por consiguiente, el lugar universal de las cosas, pero no es una sustancia, es decir, no es una entidad real. El espacio, entendido como receptáculo o lugar universal de las cosas, es - lo que Leibniz denomina en la misma correspondencia con Clarkeel espacio abstracto. En consecuencia, si bien Raffo acierta al aseverar que el dinamismo que caracteriza a los cuerpos -entendidos por Leibniz, en su juventud, como entidades sustanciales- es una condición suficiente para negar la atribución de una naturaleza sustancial al espacio, no comparto su lectura de acuerdo con la cual Leibniz abandona la tesis de la receptacularidad del espacio — como lugar universal de las cosas-.

En respuesta a una acusación de circularidad que Clarke ve en la definición leibniziana del espacio como situación en la que se sitúan los cuerpos, ${ }^{39}$ Leibniz, al introducir y explicar la noción de espacio abstracto, excluye el concepto de cuerpo como componente definicional del espacio, que consiste en un orden de situaciones. En palabras de Leibniz:

Yo no digo que el espacio sea un orden o situación que haga que las cosas puedan situarse [qui rend les choses situables] [...] Yo no digo [...] que el espacio sea un orden o situación, sino un orden de situaciones, u [orden] según el cual las situaciones se ordenan [les situations sont rangées], y

${ }^{38}$ Cfr. supra n. 6 .

${ }^{39}$ Clarke's vierte Entgegnung, § 41, GP VII, 387-388.

Revista de Filosofía Diánoia, vol. 64, no. 83 (noviembre de 2019-abril de 2020) e-ISSN: 1870-4913 • DOI: https://doi.org/10.22201/iifs.18704913e.2019.83.1718 
que el espacio abstracto es este orden de situaciones, concebidas como posibles. Así, el espacio resulta entonces ideal. ${ }^{40}$

El espacio abstracto, concebido como un orden de situaciones, no depende de los cuerpos materiales. ${ }^{41}$ Por eso, en oposición a lo que Leibniz concibe como espacio actual o real —es decir, el espacio cuya extensión coincide con la extensión de los cuerpos materiales-, el espacio abstracto es puramente ideal. Leibniz no niega, en definitiva, la posibilidad del espacio tout court al margen de los cuerpos materiales, sino que niega sólo la existencia del espacio actual fuera de la relación u orden de coexistencia o simultaneidad entre cuerpos existentes. Por ello, conviene precisar que sólo en cuanto que actual el espacio depende de los cuerpos existentes: en su posibilidad pura, el espacio no se define en relación con ellos. Raffo parece no prestar atención a esta distinción. ${ }^{42}$

La distinción entre espacio actual o real y espacio abstracto o ideal está respaldada también por otros pasajes de la correspondencia con Clarke. Uno de ellos es el siguiente:

Si no hubiese creaturas, no habría tiempo ni lugar y, por consiguiente, tampoco espacio actual [...] Yo no admito [...] que, si sólo existiese Dios, habría tiempo y lugar tal como los hay en el presente [comme à présent]. En lugar de ello, en mi opinión, ellos [el tiempo y el lugar] no estarían sino en las ideas, como simples posibilidades. ${ }^{43}$

${ }^{40}$ Leibniz' fünftes Schreiben, § 104, GP VII, 415. Arthur 2013, p. 6, considera que, por espacio abstracto, Leibniz describe la concepción newtoniana del espacio, interpretación de la que, desde luego, me alejo.

${ }^{41}$ Es la tesis que, muy acertadamente, defiende Anfray 2014 contra la lectura de Khamara 1993, p. 478, quien — tal como Vailati 1997, p. 117- sostiene que la existencia de los cuerpos materiales es una condición necesaria para la existencia del espacio (reduccionismo débil). La crítica de Anfray puede hacerse extensiva, $a$ fortiori, a Futch 2008, pp. 47-51, quien considera que la existencia de los cuerpos es una condición suficiente para el espacio (reduccionismo fuerte). En este sentido, la crítica de Anfray es solidaria con las interpretaciones "idealistas" del concepto de espacio en Leibniz que defienden con suma penetración analítica y solvencia textual Gueroult 1982 y De Risi 2007, pp. 551 y ss. Sobre este tema, cfr. también Silva 2018.

${ }^{42}$ Es por esto que la lectura de Raffo converge con las interpretaciones reduccionistas del concepto de espacio en Leibniz. Sobre estas interpretaciones, cfr. supra, nota precedente.

${ }^{43}$ Leibniz' fünftes Schreiben, § 106, GP VII, 415 (las cursivas son mías). Cfr. también Leibniz' viertes Schreiben, § 41, GP VII, 376-377.

Revista de Filosofía Diánoia, vol. 64, no. 83 (noviembre de 2019-abril de 2020) e-ISSN: 1870-4913 • DOI: https://doi.org/10.22201/iifs.18704913e.2019.83.1718 
En este fragmento, Leibniz no niega que sin las criaturas no habría espacio tout court, sino que no habría espacio actual, es decir, tal como lo hay en el presente y, por consiguiente, el espacio subsistiría en las ideas (de Dios) como una simple posibilidad. La posibilidad del espacio no depende de la existencia de los cuerpos. ${ }^{44}$

En concordancia con la relación asimétrica de precedencia ontológica entre el espacio y los cuerpos en los Nouveaux Essais, Leibniz declara en un plano epistemológico paralelo que el espacio "es una relación [rapport], un orden no solamente entre los existentes, sino que incluso entre todos los posibles como si existiesen [comme s'ils existaient] [...] $\mathrm{Su}$ verdad y realidad está fundada en Dios, como todas las verdades eternas". ${ }^{45}$ El espacio no se reduce, en definitiva, a una relación entre cuerpos existentes, pues, al concebirse como una verdad eterna, "es un indicio [marque] de las posibilidades más allá de la suposición de las existencias [...] $<\mathrm{y}>$ comprende [regarde] tanto lo posible como lo existente", ${ }^{46}$ caracterización que, no obstante, Leibniz no usa en la correspondencia con Clarke. ${ }^{47}$

La caracterización del espacio como una verdad eterna no es la única omisión de Leibniz en la correspondencia con Clarke. Existe un concepto que, siendo el correlato matemático del espacio metafísico-abstracto, Leibniz prefiere también, en el tenor de la polémica con Newton-Clarke, desestimar. Dicho concepto es el de espacio absoluto. Tal como el espacio abstracto, el espacio absoluto es la contraparte matemática del espacio actual definido por las relaciones entre los cuerpos materiales

${ }^{44}$ Siguiendo la lectura propuesta por Gueroult 1982, el pasaje parece sugerir una identificación entre el espacio como extensión geométrica (extensio) y orden entre los posibles (spatium). A la luz de diversos ángulos, Belaval 1962, pp. 222226, 1976, pp. 206-216, y 1995, pp. 165-167, parece interpretar también de esta manera el concepto leibniziano de espacio. Para una discusión de las dificultades en este asunto, las cuales remontan al carácter dual del concepto de espacio en Leibniz (lógico-formal y ontológico-material), cfr. Silva 2017, pp. 39-44. En cierto sentido, esta dualidad conceptual es paralela a —aunque independiente de-la tensión que Russell 1937, pp. 138-153, advierte entre una concepción subjetiva y otra objetiva del espacio (y del tiempo), la que ha sido, no obstante, sólidamente rebatida, por ejemplo, por Fichant 2016.

${ }^{45}$ Nouveaux Essais sur l'entendement humain, II, xiii, § 17, A VI, 6, 149.

${ }^{46}$ Nouveaux Essais sur l'entendement humain, II, xiv, § 26, A VI, 6, 154. Para un análisis in extenso de esta cuestión y otras afines, cfr. Ruiz 2014, pp. 370-425.

${ }^{47} \mathrm{Sin}$ embargo, en un borrador en el que Leibniz consigna algunas notas complementarias y posteriores a su Cuarto escrito a Clarke, sostiene que "el tiempo y el espacio pertenecen a las esencias y no a las existencias" (R, 101), descripción correlativa a la del espacio como una verdad eterna. 
existentes y equivale a la posibilidad abstracta y puramente esencial e ideal de la coexistencia. Este concepto permite asociar la teoría del espacio forjada por Leibniz en su madurez con la imagen de un receptáculo o lugar universal de las cosas.

3. La concepción leibniziana de un espacio absoluto como posibilidad de coexistencia y lugar universal de las cosas. Textos matemáticos tardíos

Atribuir a Leibniz una concepción de un espacio absoluto sería una especie de "herejía hermenéutica" si no fuese por la monumental y antológica investigación de Vincenzo de Risi. Gracias al apéndice de su obra Geometry and Monadology: Leibniz's Analysis Situs and Philosophy of Space (2007), donde publica una selección de escritos inéditos de Leibniz relativos a su filosofía del espacio, tenemos hoy una mejor comprensión de ésta. Uno de los conceptos centrales que destaca De Risi en el último periodo de las investigaciones matemáticas de Leibniz es el de espacio absoluto. Diversos pasajes de los escritos de Leibniz publicados por De Risi dan cuenta de la importancia y significado que éste confirió a ese concepto "ajeno".

En varios de estos textos inéditos, Leibniz define el concepto de espacio - a veces con el calificativo de "absoluto"- a partir del concepto de lugar, y éste a partir del concepto de situación (situs). Por ejemplo, en un manuscrito de 1695, Leibniz define el espacio como un "continuo que está constituido por todos los lugares"48 y el lugar como aquello "constituido por las situaciones [situm] mismas que están en él". ${ }^{49}$ A su vez, las situaciones se definen por los puntos que, sin ser ellos mismos extensos, hacen que la situación sí lo sea, con lo que se define la extensión por la coexistencia. ${ }^{50}$ A partir de ahí, Leibniz define el espacio como el "lugar de todos los puntos", ${ }^{51}$ que él denomina "espacio universal"52 o alternativamente —como en un opúsculo de 1712 - "espacio integral". ${ }^{53}$ Por su parte, "el punto es aquello que se da en la magnitud, y que no contiene otra cosa". ${ }^{54}$ De ahí que, "si algo se da en el punto,

${ }^{48}$ De Risi 2007, appendix, p. 587.

${ }^{49}$ De Risi 2007, appendix, p. 587.

${ }^{50}$ Cfr. De Risi 2007, appendix, p. 588. Cfr. también Initia rerum mathematicarum metaphysica, GM VII, 18 y 25.

${ }^{51}$ De Risi 2007, appendix, p. 588.

${ }^{52}$ De Risi 2007, appendix, p. 588.

${ }^{53}$ De Risi 2007, appendix, p. 596.

${ }^{54}$ De Risi 2007, appendix, p. 596.

Revista de Filosofía Diánoia, vol. 64, no. 83 (noviembre de 2019-abril de 2020) e-ISSN: 1870-4913 • DOI: https://doi.org/10.22201/iifs.18704913e.2019.83.1718 
no es sino el punto mismo", 55 de manera que "el punto es el lugar que no contiene ningún otro lugar", 56 mientras que "el espacio es el lugar que contiene todos los lugares". ${ }^{57}$ En otros términos, si "el punto es el lugar más simple", 58 "el espacio es el lugar más amplio". ${ }^{59}$

La oposición entre el espacio y el punto descrita por este conjunto de manuscritos pertenecientes al periodo tardío de la filosofía de Leibniz fue, sin embargo, concebida por él con plena claridad antes de 1690. En efecto, en un opúsculo que data de 1685, Leibniz confiesa que, al meditar con respecto a todos los objetos de la geometría, dos cuestiones resaltan manifiestamente, a saber:

El espacio absoluto mismo, en el cual no puede considerarse nada más que la extensión $[y]$ el punto, en el cual no puede considerarse nada más que la situación [situs]. El espacio no tiene situación [situm] y el punto no tiene extensión. El espacio es infinito y el punto es indivisible. El espacio es el lugar de todos los puntos. ${ }^{60}$

Espacio y punto son los dos extremos conceptuales de los elementos constitutivos de la geometría: debido a su infinitud, nada rebasa el espacio absoluto y, debido a su simplicidad, nada puede estar contenido en el punto. En otras palabras, si "el punto es lo más simple en la situación y, por lo mismo, es el lugar en el que no hay ningún otro lugar": 61

El espacio absoluto indeterminado [Spatium absolutum interminatum] es aquel en el cual la situación [situ] es la más amplia posible [amplissimum]. En otros términos, todos los lugares [locus] están en el espacio absoluto. El espacio absoluto es continuo, mientras que ningún lugar que puede ser puesto ahí lo es. El espacio absoluto es uniforme; de ahí que pueda recibir diversas determinaciones, ninguna de las cuales puede ser tan amplia [como el espacio absoluto]. ${ }^{62}$

${ }^{55}$ De Risi 2007, appendix, p. 596.

${ }^{56}$ De Risi 2007, appendix, p. 616.

${ }^{57}$ De Risi 2007, appendix, p. 616. O —como sostiene Leibniz en Initia rerum mathematicarum metaphysica - "el espacio absoluto es el lugar más pleno, es decir, el lugar de todos los lugares" (GM VII, 21).

${ }^{58}$ De Risi 2007, appendix, p. 614.

${ }^{59}$ De Risi 2007, appendix, p. 614.

${ }^{60}$ De Risi 2007, appendix, p. 624.

${ }^{61}$ De Risi 2007, appendix, p. 609.

${ }^{62}$ De Risi 2007, appendix, p. 609.

Revista de Filosofía Diánoia, vol. 64, no. 83 (noviembre de 2019-abril de 2020) e-ISSN: 1870-4913 • DOI: https://doi.org/10.22201/iifs.18704913e.2019.83.1718 
El espacio absoluto contiene todos los lugares cuya situación es la más amplia concebible y corresponde a la primera entidad extensa (ens primo extenso) o primer sujeto (proton dektikón) de la extensión, expresiones con las que Leibniz define transversalmente, a lo largo de su vida, el concepto de espacio. ${ }^{63}$ Debido a su indeterminación, continuidad y uniformidad $-\mathrm{O}$, en una palabra, debido a su idealidad-, el espacio absoluto no depende ni puede depender de los cuerpos. El espacio absoluto es el espacio metafísico-abstracto matematizable. Por ende, los cuerpos, cuya extensión coincide con la del espacio real - supuesta la inexistencia del vacío- están contenidos en el espacio absoluto, el cual representa entonces su receptáculo o lugar universal.

Ahora bien, la precedencia del espacio absoluto con respecto a los cuerpos no es una relación asimétrica infundada. En rigor, la definición del orden de esta relación no es un mero supuesto ya que, no es porque sea absoluto que el espacio preceda ontológicamente a los cuerpos, sirviéndoles de receptáculo: la razón última de esta precedencia radica en la idealidad del espacio absoluto por su infinitud. Dicho de otro modo, es porque las entidades infinitas e ideales no pueden depender de las entidades reales que el espacio absoluto, abstracto e ideal debe preceder al espacio actual, real y existente, definido por las relaciones de coexistencia entre los cuerpos materiales: ${ }^{64}$ es porque "el verdadero infinito no está sino en el absoluto, que no está formado por la adición de partes"65 que el espacio absoluto debe preceder la existencia de los cuerpos, de modo que, a contrario, "uno se equivoca queriendo imaginar un espacio absoluto que sea un todo infinito compuesto de partes": ${ }^{6}$ "no hay nada como eso[:] ésa es una noción que implica una contradicción". ${ }^{67}$ El espacio subsiste independientemente de los cuerpos. $^{68}$

En definitiva, es porque lo ideal precede a lo real que el espacio absoluto, abstracto e ideal, receptáculo de los cuerpos y lugar univer-

${ }^{63}$ Cfr., respectivamente, Leibniz a Thomasius, A II, 1, 21-22 y Entretien de Philarete et d'Ariste, GP VI, 585.

${ }^{64}$ Sobre esta cuestión, cfr. de Risi 2007, pp. 551 y ss.

${ }^{65}$ Nouveaux Essais sur l'entendement humain, II, xvii, § 1, A VI, 6, 157.

${ }^{66}$ Nouveaux Essais sur l'entendement humain, II, xvii, § 3, A VI, 6, 158. Cfr. también Leibniz a De Volder, 11 de octubre 1705, GP II, 278-279.

${ }^{67} \mathrm{Ibid}$. Cfr. también Quelques remarques sur le livre de M. Locke, A VI, 6, 7. Para un análisis de este detalle, cfr. Hartz y Cover 1988, p. 497.

${ }^{68}$ Un pasaje representativo que sintetiza las tesis que definen la concepción del espacio de Leibniz se encuentra en las Remarques sur les objections de M. Foucher, GP IV, 491-492. 
sal de las cosas, precede a los cuerpos mismos y a sus relaciones de coexistencia: éstas no definen sino la actualidad, realidad o existencia del espacio, pero no su posibilidad. El espacio es el lugar universal de las cosas porque la idealidad de la posibilidad del espacio precede a la realidad de su existencia: "lo real no deja de gobernarse perfectamente por lo ideal y lo abstracto". ${ }^{69}$

\section{Observaciones finales}

Tres son las observaciones finales que quisiera destacar. La primera es que, como consta a partir de los escritos que he analizado y en contra de la lectura de Raffo, no existe ninguna evidencia de que, después de 1671, Leibniz abandone una concepción receptacular del espacio entendida como lugar universal de las cosas: por el contrario, esta concepción está presente de manera ininterrumpida en la evolución de su filosofía del espacio.

En concordancia con la anterior, mi segunda observación apunta a la necesidad de subrayar la distinción entre receptacularidad y sustancialidad del espacio. En cierto sentido, es porque Raffo las considera equivalentes que, con todo rigor lógico, él interpreta que el abandono de la tesis de la receptacularidad es una consecuencia del abandono del sustancialismo. Sin embargo, y como he mostrado, si bien Leibniz abandona la segunda, preserva la primera. Receptacularidad y sustancialidad del espacio son dos conceptos que deben distinguirse.

Por último, la tercera observación que conviene destacar es que -lo que Raffo denomina - las concepciones estática y dinámica del espacio de Leibniz y que corresponden a lo que Leibniz denomina "espacio absoluto" o "espacio abstracto", y "espacio real" o "espacio actual" respectivamente, son perfectamente compatibles. A diferencia de lo que piensa Raffo, no parece haber entre ambas una tensión; muy por el contrario, se complementan y reflejan, de cierta manera, la fuerte influencia platónica en la metafísica del espacio de Leibniz. ${ }^{70}$

${ }^{69}$ Leibniz a Varignon, 2 de febrero de 1702, GM IV, 93. A mi juicio, al sugerir la relación de precedencia de lo abstracto o ideal por sobre lo concreto o real, esta afirmación respalda de manera indirecta la precedencia del espacio absoluto, abstracto e ideal sobre el espacio real, existente y actual, con lo que respondo a una crítica de un árbitro, quien ha objetado, en la versión previa de este artículo, esta subordinación.

${ }^{70}$ Esta discusión es un resultado parcial de la actual investigación doctoral del autor realizada en la Escuela Normal Superior de París bajo la dirección de Jean- 


\section{BIBLIOGRAFÍA}

Anfray, J.-P., 2014, "Leibniz et l'espace comme ordre général des situations correspondance avec Clarke, Cinquième écrit, § 47", en L. Peterschmitt (comp.), Espace et métaphysique de Gassendi à Kant. Anthologie, Hermann, París, pp. 271-286.

Arthur, R.T.W., 2013, "Leibniz's Theory of Space", Foundations of Science, vol. 18, no. 3, pp. 499-528.

Belaval, Y., 1962, Leibniz. Initiation à sa philosophie, Vrin, París.

—_, 1976, Études leibniziennes. De Leibniz à Hegel, Gallimard, París.

—_, 1995, Leibniz. De l'âge classique aux lumières, Lectures leibniziennes, Beauchesne, París.

De Risi, V., 2007, Geometry and Monadology. Leibniz's Analysis Situs and Philosophy of Space, Birkhäuser, Basilea.

Fichant, M., 2016, "Idéalité de la monade et réalité de la 'monadologie' : sur un lieu commun de l'interprétation”, Les Études Philosophiques, vol. 164, no. 4, pp. 515-536.

Futch, M., 2008, Leibniz's Metaphysics of Time and Space, Springer, Dordrecht.

Gueroult, M., 1982, "Space, Point and Void in Leibniz's Philosophy", en M. Hooker (comp.), Leibniz. Critical and Interpretative Essays, University of Minnesota Press, Mineápolis, pp. 284-301.

Hartz, G. y J. Cover, 1988, "Space and Time in the Leibnizian Metaphysic", Noûs, vol. 22, pp. 493-519.

Khamara, E.J., 1993, “Leibniz' Theory of Space: A Reconstruction”, The Philosophical Quarterly, vol. 43, no. 173, pp. 472-488.

— 2006, Space, Time, and Theology in the Leibniz-Newton Controversy, Ontos, Fráncfort del Meno.

Leibniz, G.W., 1849-1863, Mathematische Schriften (GM), ed. C.I. Gerhardt y H.W. Schmidt, A. Asher, Berlín, 7 vols. [reimp.: Hildesheim, Berlín/Halle, 1872].

— 1875-1890, Philosophischen Schriften (GP), ed. C.I. Gerhardt, A. Asher, Berlín, 7 vols. [reimpr.: Hildesheim, Berlín 1960-1961].

—_, 1923-, Sämtliche Schriften und Briefe (A), ed. Berlin-Brandenburgische Akademie der Wissenschaften et al., Akademie, Darmstadt/Leipzig/Berlín.

—, 1957, Correspondance Leibniz-Clarke (R), ed. A. Robinet, Presses Universitaires de France, París.

Raffo, F., 2017, “La noción de 'espacio' en los escritos juveniles de Leibniz", Diánoia, vol. 62, no. 78, pp. 75-97. DoI: 10.21898/dia.v62i78.148.

Rodriguez-Pereyra, G., 2014, Leibniz's Principle of Identity of Indiscernibles, Oxford University Press, Oxford.

Ruiz, L., 2014, El concepto leibniziano de espacio. La polémica con Clarke y el newtonianismo, Ediciones Universidad de Navarra, Pamplona.

Pascal Anfray. El autor agradece al programa CONICYT de Becas Chile, por cuyo medio se han podido financiar sus estudios doctorales.

Revista de Filosofía Diánoia, vol. 64, no. 83 (noviembre de 2019-abril de 2020)

e-ISSN: 1870-4913 • DOI: https://doi.org/10.22201/iifs.18704913e.2019.83.1718 
Russell, B., 1937, A Critical Exposition of Leibniz Philosophy, 2a. ed., Routledge, Londres.

Silva, C., 2017, "Leibniz contre l'accidentalité de l'espace et la substantialité du temps dans les Écrits à Clarke. Inhérence-persistance, coexistence-succession et synchronie-diachronie", Scripta Philosophiae Naturalis, vol. 12, pp. 27-46.

_, 2018 , "El 'pentalema' de Leibniz contra el espacio absoluto de Newton: supuestos, consecuencias y dificultades del § 5 del Tercer escrito a Clarke", Aporía, no. 15, pp. 20-41.

Vailati, E., 1997, Leibniz and Clarke. A Study of Their Correspondence, Oxford University Press, Nueva York.

Winterbourne, A.T., 1982, "On the Metaphysics of Leibnizian Space and Time", Studies in History and Philosophy of Science, vol. 13, no. 3, pp. 201-214.

Recibido el 16 de junio de 2018; revisado el 16 de enero de 2019; aceptado el 4 de marzo de 2019. 